\title{
Activated Mature Cytotoxic T-Lymphocyte
}

National Cancer Institute

\section{Source}

National Cancer Institute. Activated Mature Cytotoxic T-Lymphocyte. NCI Thesaurus.

Code C39687.

A white blood cell that is derived from a lymphocyte stem cell matured in the thymus and characterized by a CD8 marker on the surface and an antigen-specific T cell receptor which recognizes antigens in the context of MHC class I. 